\title{
An Enhanced Approach to Despeckle SAR Images
}

\author{
Duraipakkiam ANANDHI, Shanmugam VALLI \\ Dept. of Computer Science and Engineering, College of Engineering Guindy, Anna University, Chennai- 600025, \\ Tamilnadu, India \\ anandhisundar85@gmail.com,valli@annauniv.edu \\ Submitted November 1, 2017 / Accepted May 17, 2018
}

\begin{abstract}
Synthetic Aperture Radar (SAR) image processing plays a vital role in observing the earth and in understanding its varied features. A SAR image contains edges and shapes hidden by speckle noise. Therefore, despeckling is essential for subsequent feature extraction and classification. This paper presents a new despeckling method based on Non-Subsampled Contourlet Transform (NSCT) and Bayesian Maximum A Posterior (BMAP) estimation. NSCT effectively captures the SAR image features as multi-scale and multidirectional information. BMAP is a point estimation based on statistical prior distribution. So, BMAP estimation represents the aggregate behavior in each direction of the NSCT neighborhood coefficients using the statistical prior models. The dependency relationship of NSCT neighborhood coefficients by the statistical priors and $B M A P$ of point estimation shrinks the speckle noise coefficients. In this work, the NSCT higher frequency coefficients are de-speckled, since higher frequency coefficients contain more detail and more noise. This despeckling method is compared with the state-of-the-art methods using a set of reference and non-referenced quality metrics. Experimental results show that this developed method is superior to the other methods used for preserving information and for eliminating speckle noise.
\end{abstract}

\section{Keywords}

Bayesian MAP estimation, despeckling, NonSubsampled Contourlet Transform (NSCT), nonreference quality metrics, statistical models, Synthetic Aperture Radar (SAR)

\section{Introduction}

Image restoration is a challenging domain in the field of image processing. It is a process in which the original image is recovered from a noisy image. A sub-field of image processing is satellite imagery where SAR images are obtained from SAR sensor that is fixed on the satellites and aircraft. It captures high resolution images of the broader areas of the earth surface. SAR images are formed by the consistent interaction of the emitted microwave radiation with the target areas. This consistent interaction originates arbitrary constructive and destructive noisiness resulting in multiplicative noise known as speckled noise image. So, SAR images inherently contain random pixel to pixel multiplicative speckle noise [1]. There are many despeckling techniques and filters available to handle speckled SAR images by preserving the major information in the image like edge, boundary and objects.

\section{Related Works}

To remove the noise, standard SAR image restoration techniques such as Lee, Gamma map, Frost and other techniques exist [2]. But these standard spatial filters process the pixels using window selection, which leads to overlapping of pixels, exhibits limitations in preserving the features of the SAR noised images. To achieve good despeckling, Wavelet Transform (WT)-based multi-resolution techniques are used in SAR despeckling [3]. Due to lack of directionality, WT does not capture the high-dimensional distinctive information in SAR images. So multi directional transforms are used to effectively capture the directional geometric features of SAR images. Contourlet Transform (CT) provides multi-scale, multi-directional geometric analysis in which directional information gathering allows good despeckling in SAR images [4]. But CT incurs dithering distortion due to discrete subsampling. Therefore, CT despeckling results in loss of information in SAR images. NSCT is a translation invariant version of the CT [5]. NSCT inherits the improved features of CT and preserves abundance of details and directional information in the SAR images. Therefore NSCT is used for SAR despeckling to achieve good preservation of features.

Several SAR despeckling methods have been developed based on the threshold criterion in the NSCT domain. Self-adaptive threshold [6], estimation of speckle variance [7], threshold shrinkage [8] and morphological operators [9] are some of the NSCT despeckling approaches. These methods are based on the single value thresholding function. Selection of threshold value in satellite images is not an easy task as satellite images have low contrast. Selection of small threshold value causes resultant image to be noisy, while resultant image at the edges and boundaries gets blurred for a large threshold value. So thresholding scheme 
is not sufficient to cover all the valuable information of the images which leads to loss of information in the resultant de-speckled images. To obtain the sufficient smoothening and retain the maximum features, MMSE and MAP Bayesian despeckling approaches are found to be attractive. These make use of clutter statistics for accurate texture estimation instead of single threshold value based on image property or the empirical values based on fixed spatial filters [10].

The MMSE-based filters perform despeckling by minimizing the mean square error between the clutter and the estimated texture. However, these restoration filters over smoothen the images, leading to loss of textural information. On the contrary, the MAP-based approach overcomes the limitation of MMSE filters by utilizing prior knowledge about statistical distribution of texture and speckle individually. In the past, lots of Gaussian distribution based MAP estimation in WT domain is used for SAR despeckling to achieve good noise reduction and feature preservation [11], [12]. But WT is not sufficient for edge preservation as it lacks higher frequency information. So in this work NSCT is used to create better analysis of texture of an image [13]. But NSCT mainly handles the additive noise pattern [14]. NSCT processing converts the multiplicative noise to additive noise. But this nonlinear transform completely changes the statistics of the speckle model and also affects the quality of the image. Any transforms such as WT, NSCT follows the generalized Gaussian distribution. Gaussian distribution is commonly used for additive noise model. Due to the multiplicative noise background of the SAR image, NSCT with Gaussian statistical prior models are not appropriate for despeckling the SAR images.

To obtain good noise-free and edge-preserved SAR images, this work uses non-Gaussian NSCT model coefficients using Rayleigh and Laplacian statistical prior distributions [15]. The main contributions of this proposed work are stated as follows:

- Modelling the NSCT higher frequency coefficients using Rayleigh distribution to preserve the multiplicative noise pattern and Laplacian distribution is used to preserve maximum features.

- BMAP based on the statistical measure of Rayleigh and Laplacian distributions is used to effectively eliminate the noisy coefficients.

This proposed NSCT-BMAP approach is compared with state of art Bayesian SAR despeckling techniques such as Generalized Guided Filter with Bayesian Non Local Means (GGF-BNLM) [16], Cauchy exponential compound Gaussian (CE-CG) maximum a posterior (MAP) [17] and Homomorphic Bayesian in terms of Bayes shrink in discrete WT (HBSWT) [18] and Non-Subsampled contourlet Gaussian Laplacian (NSCT-GL) MAP [19] approaches.

The rest of this paper is structured as follows: Section 3 explains this work. Section 4 describes the quality metrics.
Section 5 discusses the experimental results and Section 6 concludes this work.

\section{Despeckling Approach}

The idea of this work is to correctly define the noise and noise free content using prior distributions which is appropriate for the statistics of the SAR image model which contains random noise. Speckle noise is randomly mixed with surface texture information of the image. So the statistics of the speckle image model is identified by the multiplicative noise pattern. The main challenge in the modelling of the SAR image is the selection of the statistical prior model that decides the accuracy of the resultant image. If the prior model is chosen properly the noise can be removed effectively. Wavelets or wavelet extensions such as CT and NSCT transforms, mainly handle the additive noisy pattern [19]. It converts the multiplicative noise to additive noise. But, this nonlinear transform completely changes the statistics of the speckle model and also affects the quality of the image. The prior models of SAR NSCT coefficients are based on the Gaussian distribution. Gaussian distribution is an additive model. Due to the multiplicative background of the SAR image, NSCT with Gaussian statistical prior models are not appropriate for despeckling the SAR images. The histogram of the SAR image model with speckle noise is shown in Fig. 1 that is Rayleigh like model that does not follow the Gaussian bell shaped model. The Rayleigh model accurately describes the statistics of an SAR image.

This work uses Rayleigh $(R)$ and Laplacian $(L)$ distribution-based statistical priors to model the noise and noisefree coefficients for despeckling SAR images in the NSCT domain. NSCT SAR additive noise coefficients are converted to multiplicative noise content using $R$ distribution. $L$ distribution serves as the local variance operator. Statistical estimation is carried out on the $R L$ distributions of multi-scale and multi-directional NSCT coefficients. BMAP is a point estimation in which most often values are estimated to eliminate the noise. Based on the $R L$ statistical information, BMAP removes the noisy coefficients. In this work,

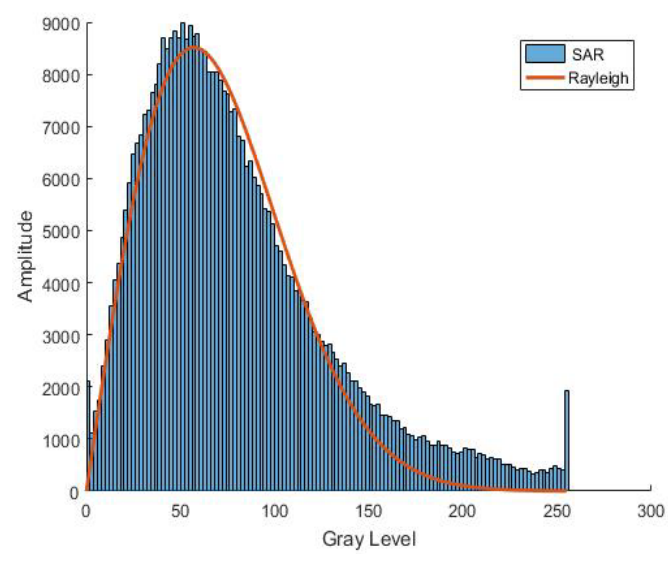

Fig. 1. SAR image statistics and its Rayleigh distribution. 


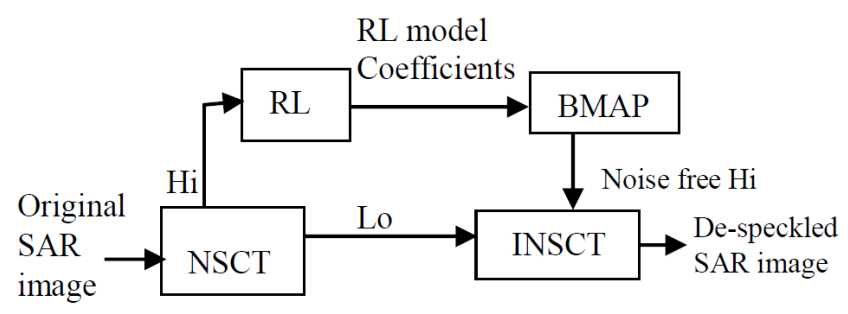

Fig. 2. The frame work of the proposed NSCT-BMAP despeckling method.

NSCT transform is processed up to 3 levels which delivers different resolution ranges of lower and higher frequency coefficients. Lower frequency $(L o)$ coefficients are contours that contain the magnitude of an image. Similarly, higher frequency $(\mathrm{Hi})$ coefficients are regions that contain major information on features such as shapes, edges and textures. Thus, higher frequency coefficients have more noise. Accordingly, this BMAP despeckling work concentrates only on the higher frequency coefficients. Initially, NSCT is applied on the noisy SAR images to get the lower and higher frequency coefficients. Second, Rayleigh $(R)$ and Laplacian $(L)$ distributions are applied on the NSCT higher frequency coefficients. Third, Bayesian MAP is applied on the RL model coefficients. Lastly, Inverse NonSubsampled Contourlet Transform (INSCT) is applied on the lower and higher frequency coefficients to obtain the de-speckled SAR image. The entire work is referred to as the NSCT-BMAP despeckling method. The block diagram of this NSCT-BMAP despeckling is depicted in Fig. 2. NSCT and BMAP estimation are explained in the following section.

\subsection{NSCT-BMAP}

NSCT is a combination of Non Subsampled Pyramid (NSP) and Non Subsampled Directional Filter Banks (NSDFB). NSP scatters the resolution singularity and NSDFB distinguishes the higher dimensional resolution singularity. Therefore, NSCT offers better frequency selectivity and regularity. BMAP is an estimation of the values that appears most often in a set of data of the random event, where the conditional probability is allocated after the relevant evidence is taken into account. Rayleigh and Laplacian probability density functions model the noise and noise free coefficients respectively. Statistical information of each model's coefficient attains posterior probability conditions. Based on the conditional probability, noisy coefficients are eliminated. RL-based BMAP is explained as follows:

The observed SAR image of the higher frequency coefficients is given in (1).

$$
\text { Original }_{\mathrm{SAR}}=R+L \text {. }
$$

Equation (1) is rewritten as (2)

$$
L=\text { Original }_{\mathrm{SAR}}-R \text {. }
$$

In (1) and (2) $R$ represents the noisy component and $L$ represents the noise-free component. They are defined in (3) and (5) respectively.

$$
R=\frac{A^{2}}{\alpha^{2}} \exp \left(\frac{-A^{2}}{2 \alpha^{2}}\right)
$$

where $A$ is the noise amplitude and $\alpha$ is the fading parameter. $\alpha$ is defined in (4).

$$
\alpha=\frac{\sigma_{\mathrm{A}}}{\sqrt{2}}
$$

where $\sigma_{\mathrm{A}}$ is the noisy variance.

$$
L=\frac{1}{\sigma_{K} \sqrt{2 \pi}} \exp \left(-\frac{\sqrt{2\left|K-\mu_{K}\right|}}{\sigma_{K}}\right) .
$$

In (5), $\sigma_{K}$ is the noise-free variance and $\mu_{K}$ is the noise-free mean.

$$
\begin{gathered}
\mu_{K}=E(K), \\
\sigma_{K}=\Psi \mu_{K}^{2} C_{K}^{2}
\end{gathered}
$$

where $\mu_{K}^{2}$ and $C_{K}^{2}$ is the mean of the noise and standard deviation of noise respectively.

Bayes rule and log function is applied on (2) and results in noise free $\left(L^{\prime}\right)$ coefficients which is given in (8).

$$
L^{\prime}=\arg \max _{H i L}[p(L) \mid p(R)]
$$

Based on (8), BMAP estimator for $\mathrm{Hi}$ coefficients is obtained and given in (9).

$L^{\prime}=$

$$
\arg \max _{H i L}\left[\begin{array}{l}
\log p\left(\frac{1}{\sigma_{K} \sqrt{2 \pi}} \exp \left(-\frac{\sqrt{2\left|K-\mu_{K}\right|}}{\sigma_{K}}\right)\right) \mid l . \\
p\left(\frac{A^{2}}{\alpha^{2}} \exp \left(\frac{-A^{2}}{2 \alpha^{2}}\right)\right)
\end{array}\right]
$$

From (5), (6) and (7), a set of statistical parameters $\alpha$, $\mu_{K}$ and $\sigma_{K}$ is calculated for higher frequency coefficients. The parameters are initialized first and then a maximum posterior step is iteratively performed until the parameters meet the convergence conditions.

Equation (9) is differentiated with respect to $k$ and equated to zero which results in (10)

$$
L^{\prime}=R-\left|\frac{\sqrt{2 \alpha^{4}+4 \alpha^{4} \sigma_{k}^{2}} \pm \alpha^{2} \sqrt{2}}{2 \sigma_{K}}\right|
$$

In (11), $R-\left|\frac{\sqrt{2 \alpha^{4}+4 \alpha^{4} \sigma_{K}^{2}} \pm \alpha^{2} \sqrt{2}}{2 \sigma_{K}}\right|$ term is simplified as variable $P$. 


$$
\text { Noise free } H i \text { or } L^{\prime}=\left\{\begin{array}{l}
R-P, \text { if } R>\mu_{K}+|P| \\
R+P, \text { if } R>\mu_{K}-|P|
\end{array}\right.
$$

Variance is always a non-negative number. A small value of variance indicates that the data points tend to be very close to mean or the expected value. A higher value of variance indicates that the data points are highly spread out around the mean. Equation (11) gives the relation of mean $\mu$ and variance $\sigma$ that result in optimal $R L$ coefficients, and noise-free $H i$ coefficient. Consequently, estimated noisefree $H i$ and $L o$ coefficients are reconstructed by the INSCT to get the final SAR de-speckled image. Algorithm 1 gives the entire process of this developed NSCT-BMAP despeckling.

Algorithm 1. NSCT-BMAP despeckling

I/P: SAR image

O/P: De-speckled SAR Image

1. Application of NSCT on the SAR image to extract $H i$ and $L o$, coefficients

2. for all $\mathrm{Hi}$ do $/ / *$ Statistical estimation

3. $\quad R=\frac{A^{2}}{\alpha^{2}} \exp \left(\frac{A^{2}}{2 \alpha^{2}}\right)$

4. $\quad L=\frac{1}{\sigma_{K} \sqrt{2 \pi}} \exp \left(-\frac{\sqrt{2\left|K-\mu_{K}\right|}}{\sigma_{K}}\right)$

5. end for

6. $\quad L^{\prime}=\arg \max _{H i L}[p(L) \mid p(R)]$

6. // BMAP

7. $\quad$ if $\left(R>\mu_{K}+|P|\right)$ then

8.

$H i=R-P$

$$
\text { elseif }\left(R>\mu_{K}-|P|\right) \text { then }
$$

9.

11.

$$
H i=R+P
$$

end if

12. Application of INSCT to reconstruct the noise-free $H i$ coefficients $\left(L^{\prime}\right)$ and $L o$ coefficients to get the despeckled SAR image.

\section{Performance Measures}

A good SAR despeckling technique should have essentially four important characteristics [20]: (1) It should lead to speckle reduction in the homogeneous areas; (2) It should preserve scene features such as texture, edges, point target and so on; (3) It should preserve radiometric information; and (4) It should result in the absence of artefacts. To assess the capacity of a despeckling technique, different quality metrics are used. Two types of quality metrics are non-reference measures and full-reference measures. The non-reference measures are applied on the real SAR im- ages and the full-reference measures are applied when a reference SAR image is generated by simulation. This work considers both non-reference and reference measures, since real SAR and synthetic SAR images are used. For reference SAR image, Peak Signal Noise Ratio (PSNR), Mean Square Error (MSE), Universal Image Quality Index (UIQI) and Structural Similarity Index Measures (SSIM) are used [21]. PSNR is one of the important performance metrics in the denoising procedure. When the value of the PSNR is high, then the quality of the denoised image is good, otherwise it is considered bad. MSE measures the error between two comparing a despeckled image and reference image. Universal Image Quality Index (UIQI) helps to analyze the linear correlation, luminance and contrast of the image. SSIM is used to measure the similarity between denoised images against reference image. SSIM ranges between -1 to 1 and UIQI values ranges between 0 and 1 . Values near to 1 represent better image quality. MSE, PSNR, SSIM and UIQI are given in (11)-(14).

$$
M S E=\frac{1}{m, n}(\operatorname{Di}(m, n)-O i(m, n))
$$

where $m$ and $n$ is the number of rows and columns respectively. $O i(m, n)$ refers the original SAR image, $D i(m, n)$ refers the despeckled SAR image.

$$
\begin{gathered}
P S N R=10 \log _{10}\left(\frac{255 \times 255}{M S E}\right), \\
S S I M=\frac{\left(2 \mu_{D i} \mu_{O i}+C_{1}\right)\left(2 \sigma_{D i O i}+C_{2}\right)}{\left(\mu_{D i}^{2}+\mu_{O i}^{2}+C_{1}\right)\left(\sigma_{D i}^{2}+\sigma_{O i}^{2}+C_{2}\right)}, \\
U I Q I=\frac{\sigma_{D i O i}}{\sigma_{D i} \sigma_{O i}} \cdot \frac{2 D i O i}{D i^{2}+O i^{2}} \cdot \frac{2 \sigma_{D i} \cdot \sigma_{O i}}{\sigma_{D i}^{2}+\sigma_{O i}^{2}} .
\end{gathered}
$$

In (13) and (14), $\mu_{D i}, \mu_{O i,} \sigma_{D i,}, \sigma_{O i,}, \sigma_{D i, O i}$, are local means, standard deviation and cross variance for original and despeckled images.

The basic non-reference measures [22] considered are Noise Mean Value (NMV), Noise Variance (NV), the Mean Square Difference (MSD) and an Equivalent Number of Looks (ENL). NMV and NV are used to determine the noise content in the despeckled image. A lower value of NMV indicates better performance. MSD is the average difference of pixels between the SAR and the despeckled image. The higher value of MSD indicates that a higher amount of noise content is removed. ENL is generally used to show the smoothening effect in the despeckling methods. A higher value of ENL implies a good despeckling performance. NMV, NV, MSD, and ENL are given in (15)-(18) respectively.

$$
\begin{gathered}
N M V=\sum_{m, n} \frac{D i(m, n)}{O i(m, n)}, \\
N V=\sum_{m, n} \frac{(D i(m, n)-N M V)^{2}}{m \times n},
\end{gathered}
$$




$$
\begin{gathered}
M S D=\sum_{m, n} \frac{(O i(m, n)-D i(m, n))^{2}}{m \times n}, \\
E N L=\frac{N M V^{2}}{N V} .
\end{gathered}
$$

Edge preservation in despeckled images is calculated using the Edge Save Index (ESI), Signal to Clutter Ratio (SCR), Deflection Ratio (DR). ESI is the edge-saving ability in the horizontal and vertical directions of the despeckled image. Both the directions of ESI are known as $\mathrm{ESI}_{\mathrm{H}}$ and ESI $\mathrm{V}$ and are given by (19) and (20). SCR is used to measure the strong edge targets of the de-speckled image as shown in (21). DR measures the overall information of the despeckled image as given by (22). DR should be high when stronger reflector points are present within the pixels.

$$
\begin{gathered}
E S I_{\mathrm{H}}=\frac{\sum_{x=1}^{m} \sum_{y=1}^{n}\left|\operatorname{Di}(m, n)_{x, y+1}-\operatorname{Di}(m, n)_{x, y}\right|}{\operatorname{Oi}(m, n)_{x, y+1}-\operatorname{Oi}(m, n)_{x, y}}, \\
E S I_{\mathrm{V}}=\frac{\sum_{x=1}^{m} \sum_{y=1}^{n}\left|\operatorname{Di}(m, n)_{x, y+1}-\operatorname{Di}(m, n)_{x, y}\right|}{O i(m, n)_{x, y+1}-\operatorname{Oi}(m, n)_{x, y}}, \\
S C R=10 \log _{10} \frac{\sum_{m} \sum_{n}|\operatorname{Di}(m, n)|}{T \sigma(m, n)} .
\end{gathered}
$$

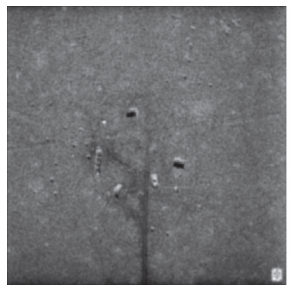

(a)

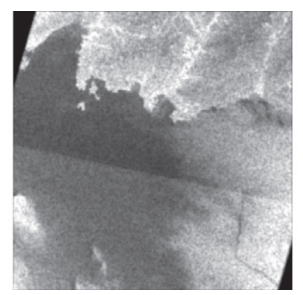

(b)
In (21), $\sigma(m, n)$ is the standard deviation of clutter target point and $T$ is the total number of point target pixels.

$$
D R=\frac{1}{m \times n} \sum_{m, n} \frac{D i(m, n)-N M V}{N V} .
$$

\section{Results and Discussion}

In this section, experimental results and their performance characteristics are explained using some synthetic and real SAR images in order to validate the efficiency of the proposed algorithm. Figure 3 shows synthetic SAR images and speckle noise is added to these images using different levels of variance ( $\sigma=4, \sigma=10, \sigma=20, \sigma=30$ and $\sigma=40$ ). The noise added SAR images are shown in Fig. 4. Figure 5 shows the sample real SAR images which contain original speckle noise obtained by sensors. Real SAR images with original size of $1500 \times 2672$ and noised SAR images with the size of $512 \times 512$ are used to verify the performance of the proposed algorithm for the qualitative and quantitative assessment. All experiments are conducted in MATLAB version R2010a on Intel(R), Core(TM), i5, CPU $2.30 \mathrm{GHz}, 4$ GB RAM and 32-bit operating system. The same system configuration is used to opinion the execution time of the proposed algorithm and the existing techniques. The experimental results of the proposed method are compared with the results of Bayesian based state of techniques GGF-BNLM [16], CE-CG [17], HBSWT [18] and NSCT- GL [19] methods.

Fig. 3. Synthetic SAR images: (a) SAR1, (b) SAR2, (c) SAR3, (d) SAR4

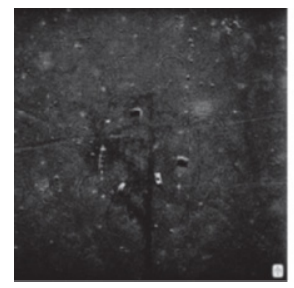

(a)

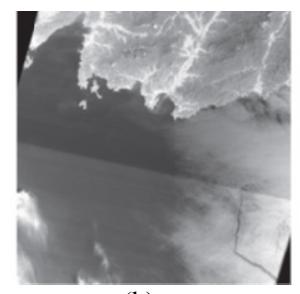

(b)

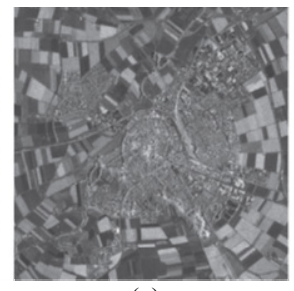

(c)

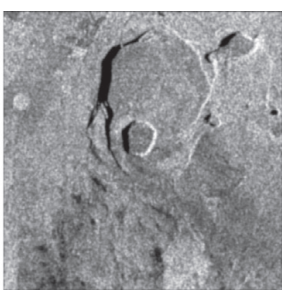

(d)

Fig. 4. Noise added SAR images: (a) SAR1, (b) SAR2, (c) SAR3, (d) SAR4.

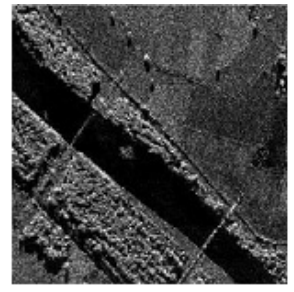

(a)

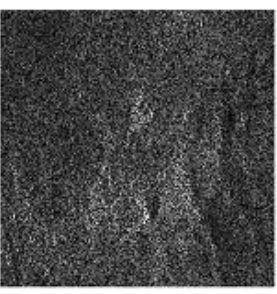

(b)

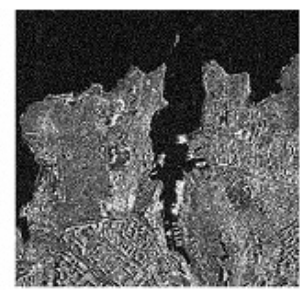

(c)

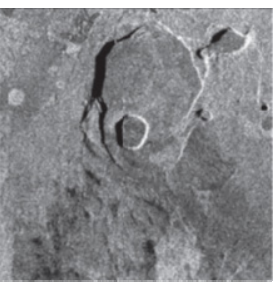

(d)

Fig. 5. Real SAR images: (a) SAR5, (b) SAR6, (c) SAR7, (d) SAR8.

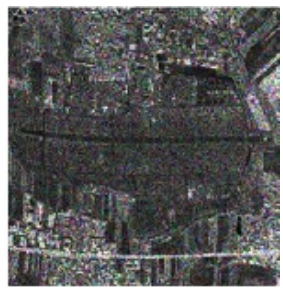

(d) 


\subsection{Datasets}

To demonstrate the effectiveness of the developed NSCT-BMAP approach, eight different SAR images are taken with different background nature. Processed SAR1 to SAR4 images are taken from the public access database NASAPHOTOJOURNAL - jet population Laboratories [23], Belgian Platform on Earth Observation [24] and Sandia National Laboratories of U.S Government, Satellite Imagery - Airbus Defense and Space [25]. The real speckled SAR images namely SAR5, SAR6, SAR7 and SAR8 as shown in Fig. 5 are taken from the European space agency (ESA) [26] database.

\subsection{Experimental Evaluation using Synthetic SAR Image}

Figures 6-10 show the results of HBSWT, GGFBNLM, CE-CG, NSCT-GL methods and the proposed method respectively. It is seen that the proposed algorithm attained good visual appearance than the other methods. The performance measures such as PSNR, SSIM, UIQI and RMSE are evaluated at different noise variances as shown in Tabs. 1 and 2. The best results are shown in bold. Table 1 shows comparison of existing and the proposed despeckling method based on PSNR and SSIM, while Table 2 shows those based on UIQI and RMSE. The results of HBSWT [18], GGF-BNLM [16], CE-CG [17] and NSCT- GL [19] methods show good performance in terms of visual appearance of image, but still presence of artifacts and slight disturbance in edges is seen. The proposed
NSCT-BMAP method shows the best results compared to the existing methods.

On applying all the methods on the SAR1 image, it is observed that at noise variance level 10, NSCT-GL method shows the best results in terms of PSNR, while in rest of the noise variances, the proposed NSCT-BMAP method shows the best results.

For the SAR2 image, the proposed NSCT-BMAP method shows the best results in terms of PSNR and SSIM. For SAR3 image, GGF-BNLM shows the better results at low variance level i.e. 4 in terms of PSNR. CE-CG shows the better results at variance level 10 in terms of SSIM, while in rest cases in terms of PSNR and SSIM, NSCTBMAP method shows the best results as depicted in Tab. 1. For SAR1 image, HBSWT method shows good result at noise level 30 in terms of UIQI. For SAR2 image, CE-CG method shows good result at variance level 10 in terms of UIQI. For SAR3 image, GGF-BNLM method shows better result at variance level 10 in terms of UIQI. In SAR4 image HBSWT method shows good result at noise variance level 20 in terms of UIQI. On comparing HBSWT, CE-CG and GGF-BNLM methods in terms of UIQI, the proposed NSCT-BMAP method is better in 95 percent of the cases while NSCT-GL shows best results in almost all the rest cases. The results of the proposed NSCT-BMAP method are far better than other methods in terms of MSE. The obtained UIQI, MSE, PSNR and SSIM values in terms of texture and edge preservation show good result using developed method than the HBSWT [18], GGF-BNLM [16], CE-CG [17] and NSCT- GL [19] methods.

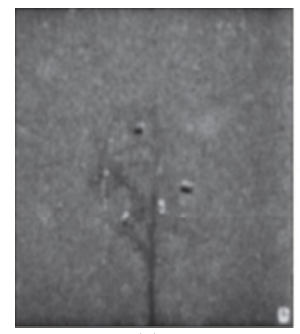

(a)

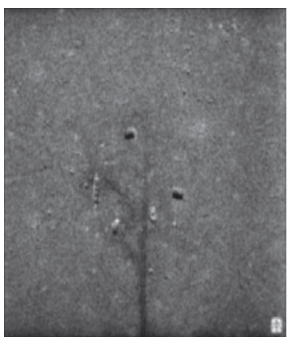

(a)

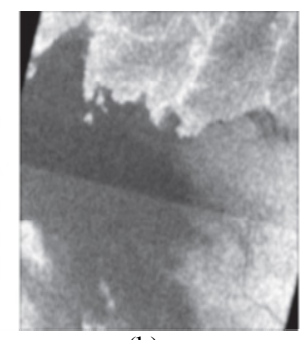

(b)

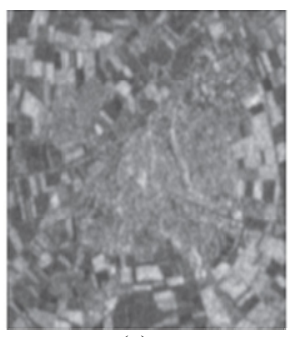

(c)

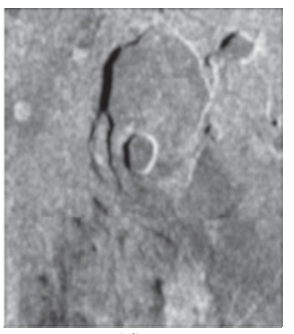

(d)

Fig. 6. Despeckled images using [18] method.

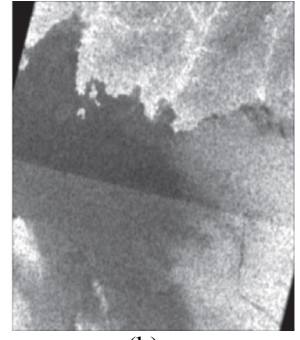

(b)

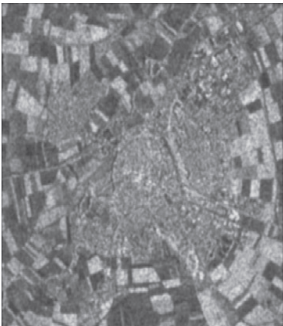

(c)

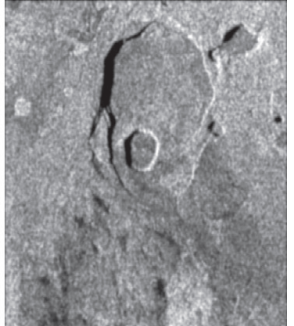

(d)

Fig. 7. Despeckled images using [16] method. 


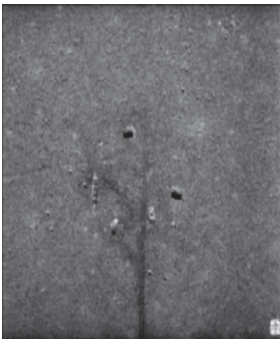

(a)

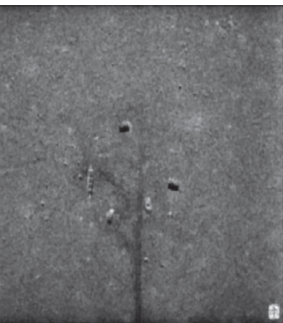

(a)

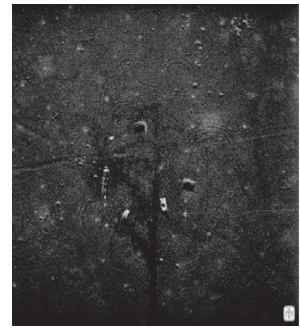

(a)

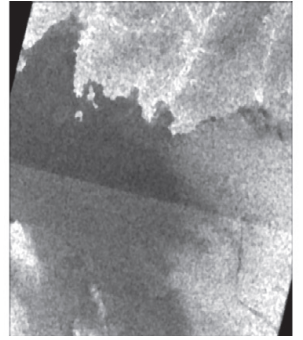

(b)

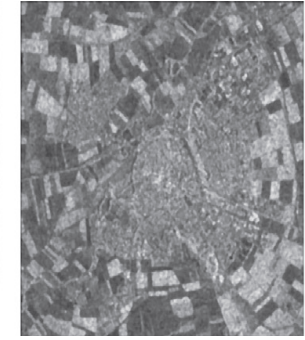

(c)

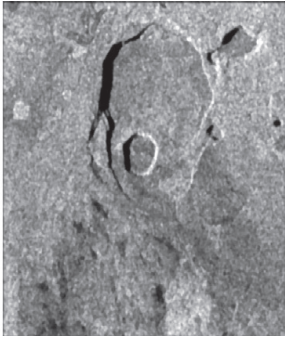

(d)

Fig. 8. Despeckled images using [17] method.

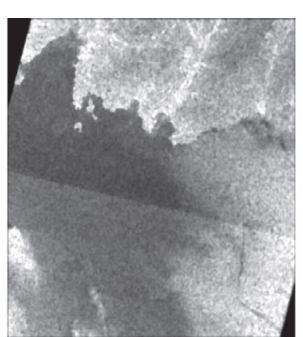

(b)

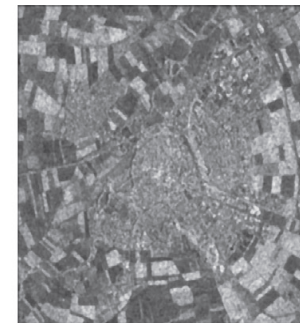

(c)

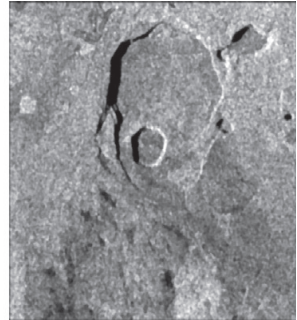

(d)

Fig. 9. Despeckled images using [19] method.

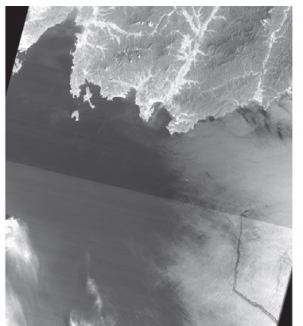

(b)

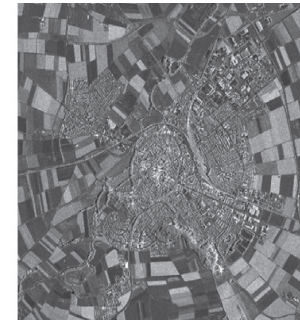

(c)

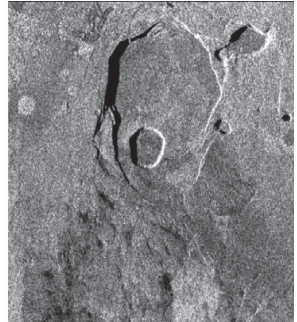

(d)

Fig. 10. Despeckled images using the proposed method.

\begin{tabular}{|c|c|c|c|c|c|c|c|c|c|c|c|}
\hline \multirow{2}{*}{ Images } & \multirow{2}{*}{ Methods } & \multicolumn{5}{|c|}{ PSNR of different noise variance } & \multicolumn{5}{|c|}{ SSIM of different noise variance } \\
\hline & & $\sigma=4$ & $\sigma=10$ & $\sigma=20$ & $\sigma=30$ & $\sigma=40$ & $\sigma=4$ & $\sigma=10$ & $\sigma=20$ & $\sigma=30$ & $\sigma=40$ \\
\hline \multirow{5}{*}{ SAR1 } & [18] & 43.7758 & 41.6010 & 39.9001 & 38.4579 & 36.6709 & 0.9725 & 0.9691 & 0.9358 & 0.9229 & 0.9209 \\
\hline & [16] & 40.9001 & 40.9901 & 38.2243 & 40.5006 & 36.7988 & 0.8034 & 0.9021 & 0.9287 & 0.8976 & 0.9013 \\
\hline & [17] & 38.4333 & 40.6235 & 40.6785 & 41.9138 & 37.9537 & 0.9675 & 0.9543 & 0.9706 & 0.8901 & 0.8671 \\
\hline & {$[19]$} & 40.8567 & 42.0561 & 39.7237 & 40.4675 & 41.0821 & 0.9718 & 0.9578 & 0.9689 & 0.9678 & 0.9799 \\
\hline & $\begin{array}{c}\text { Proposed } \\
\text { method }\end{array}$ & 43.9899 & 41.8987 & 43.9891 & 45.8119 & 45.0201 & 0.9789 & 0.9621 & 0.9788 & 0.9834 & 0.9899 \\
\hline \multirow{5}{*}{ SAR2 } & {$[18]$} & 42.7729 & 40.7504 & 37.0465 & 34.9169 & 32.8688 & 0.9749 & 0.9489 & 0.9252 & 0.9001 & 0.8914 \\
\hline & [16] & 38.0091 & 40.9341 & 38.2893 & 40.5326 & 36.7118 & 0.8004 & 0.9221 & 0.9007 & 0.9006 & 0.8993 \\
\hline & [17] & 38.4003 & 40.6125 & 39.6005 & 41.9888 & 37.9007 & 0.9577 & 0.9243 & 0.9726 & 0.9101 & 0.8881 \\
\hline & {$[19]$} & 40.8127 & 42.0111 & 39.7637 & 40.4005 & 41.0111 & 0.9678 & 0.9438 & 0.9677 & 0.9688 & 0.9299 \\
\hline & $\begin{array}{c}\text { Proposed } \\
\text { method }\end{array}$ & 42.9009 & 43.8007 & 43.9991 & 43.8911 & 43.0001 & 0.9899 & 0.9711 & 0.9898 & 0.9804 & 0.9699 \\
\hline \multirow{5}{*}{ SAR3 } & {$[18]$} & 39.2991 & 36.9472 & 34.3189 & 34.9939 & 31.1602 & 0.9808 & 0.9377 & 0.9211 & 0.9085 & 0.8957 \\
\hline & {$[16]$} & 42.8881 & 38.9975 & 36.9430 & 34.7084 & 32.8621 & 0.9731 & 0.9322 & 0.8519 & 0.8321 & 0.8902 \\
\hline & [17] & 38.0751 & 39.6741 & 39.3621 & 35.8572 & 31.0964 & 0.9764 & 0.9596 & 0.8896 & 0.8945 & 0.8911 \\
\hline & [19] & 41.0078 & 40.3301 & 38.9531 & 33.8451 & 35.6321 & 0.9889 & 0.9578 & 0.9521 & 0.9175 & 0.9117 \\
\hline & $\begin{array}{c}\text { Proposed } \\
\text { method }\end{array}$ & 41.2222 & 40.6832 & 39.0751 & 38.7541 & 36.9531 & 0.9905 & 0.9431 & 0.9476 & 0.9575 & 0.9473 \\
\hline \multirow{5}{*}{ SAR4 } & {$[18]$} & 36.9001 & 33.3331 & 31.9939 & 30.0272 & 29.9536 & 0.9040 & 0.9244 & 0.8758 & 0.8655 & 0.8529 \\
\hline & {$[16]$} & 39.1181 & 33.8905 & 32.7653 & 34.7084 & 28.0021 & 0.9021 & 0.9002 & 0.7659 & 0.8801 & 0.8882 \\
\hline & {$[17]$} & 38.0981 & 34.0041 & 32.6621 & 35.8512 & 29.9990 & 0.9224 & 0.9236 & 0.9196 & 0.8879 & 0.8541 \\
\hline & [19] & 41.0008 & 34.3331 & 34.7623 & 33.8451 & 31.8740 & 0.9492 & 0.9342 & 0.9541 & 0.9784 & 0.8643 \\
\hline & $\begin{array}{c}\text { Proposed } \\
\text { method }\end{array}$ & 42.6852 & 35.7532 & 36.8551 & 38.7571 & 34.0431 & 0.9675 & 0.9511 & 0.9753 & 0.9705 & 0.9203 \\
\hline
\end{tabular}

Tab. 1. The results of performance evaluation on synthetic SAR images using PSNR and SSIM. 


\begin{tabular}{|c|c|c|c|c|c|c|c|c|c|c|c|}
\hline \multirow{2}{*}{ Images } & \multirow{2}{*}{ Methods } & \multicolumn{5}{|c|}{ UIQI of different noise variance } & \multicolumn{5}{|c|}{ MSE of different noise variance } \\
\hline & & $\sigma=4$ & $\sigma=10$ & $\sigma=20$ & $\sigma=30$ & $\sigma=40$ & $\sigma=4$ & $\sigma=10$ & $\sigma=20$ & $\sigma=30$ & $\sigma=40$ \\
\hline \multirow{5}{*}{ SAR1 } & [18] & 0.9060 & 0.8952 & 0.8761 & 0.8956 & 0.8758 & 0.0072 & 0.0094 & 0.0127 & 0.0151 & 0.0164 \\
\hline & [16] & 0.9237 & 0.9100 & 0.7622 & 0.7981 & 0.9412 & 0.1099 & 0.0510 & 0.0275 & 0.1839 & 0.0273 \\
\hline & [17] & 0.9213 & 0.9120 & 0.7341 & 0.8431 & 0.8711 & 0.0057 & 0.0081 & 0.0338 & 0.0359 & 0.0660 \\
\hline & {$[19]$} & 0.8751 & 0.9042 & 0.8862 & 0.8651 & 0.9187 & 0.0075 & 0.0987 & 0.1170 & 0.0502 & 0.0954 \\
\hline & $\begin{array}{c}\text { Proposed } \\
\text { method }\end{array}$ & 0.9782 & 0.9333 & 0.8951 & 0.8765 & 0.9499 & 0.0028 & 0.0018 & 0.0234 & 0.0099 & 0.0111 \\
\hline \multirow{5}{*}{ SAR2 } & {$[18]$} & 0.8773 & 0.8458 & 0.8384 & 0.8399 & 0.8228 & 0.0072 & 0.0116 & 0.0157 & 0.0201 & 0.0227 \\
\hline & [16] & 0.9100 & 0.8891 & 0.8914 & 0.8341 & 0.9210 & 0.1210 & 0.1999 & 0.0673 & 0.1098 & 0.1009 \\
\hline & [17] & 0.9165 & 0.9674 & 0.9231 & 0.8061 & 0.8671 & 0.0225 & 0.1201 & 0.0567 & 0.0675 & 0.1870 \\
\hline & {$[19]$} & 0.8921 & 0.8631 & 0.8125 & 0.8143 & 0.8931 & 0.0986 & 0.0101 & 0.0177 & 0.1200 & 0.0983 \\
\hline & $\begin{array}{c}\begin{array}{c}\text { Proposed } \\
\text { method }\end{array} \\
\text {. }\end{array}$ & 0.9501 & 0.9568 & 0.9298 & 0.8486 & 0.9764 & 0.0058 & 0.0071 & 0.0130 & 0.0110 & 0.0188 \\
\hline \multirow{5}{*}{ SAR3 } & {$[18]$} & 0.9079 & 0.9016 & 0.8921 & 0.8804 & 0.8737 & 0.0153 & 0.0178 & 0.0215 & 0.0245 & 0.0277 \\
\hline & {$[16]$} & 0.8912 & 0.9414 & 0.8786 & 0.7861 & 0.7812 & 0.9771 & 0.1008 & 0.1236 & 0.1837 & 0.0566 \\
\hline & [17] & 0.8891 & 0.9341 & 0.8954 & 0.8817 & 0.7631 & 0.0986 & 0.0987 & 0.1097 & 0.1980 & 0.0410 \\
\hline & {$[19]$} & 0.9125 & 0.9145 & 0.9232 & 0.9132 & 0.8912 & 0.0180 & 0.0563 & 0.0156 & 0.0675 & 0.0450 \\
\hline & $\begin{array}{c}\text { Proposed } \\
\text { method }\end{array}$ & 0.9471 & 0.9614 & 0.9571 & 0.9451 & 0.9123 & 0.0122 & 0.0045 & 0.0089 & 0.0110 & 0.0198 \\
\hline \multirow{5}{*}{ SAR4 } & {$[18]$} & 0.8681 & 0.8648 & 0.9521 & 0.8457 & 0.8375 & 0.0218 & 0.0241 & 0.0282 & 0.0315 & 0.0348 \\
\hline & [16] & 0.9431 & 0.8876 & 0.8964 & 0.7759 & 0.8402 & 0.1880 & 0.1001 & 0.0997 & 0.1240 & 0.1830 \\
\hline & [17] & 0.9127 & 0.9108 & 0.7859 & 0.9456 & 0.8195 & 0.0531 & 0.7770 & 0.1099 & 0.1210 & 0.0999 \\
\hline & {$[19]$} & 0.8007 & 0.8976 & 0.8075 & 0.8753 & 0.8853 & 0.0675 & 0.0675 & 0.0675 & 0.0988 & 0.1231 \\
\hline & $\begin{array}{c}\text { Proposed } \\
\text { method }\end{array}$ & 0.9660 & 0.9313 & 0.9343 & 0.9568 & 0.9564 & 0.0110 & 0.0098 & 0.0175 & 0.0122 & 0.0121 \\
\hline
\end{tabular}

Tab. 2. The results of performance evaluation on synthetic SAR images using UIQI and MSE.

\subsection{Experimental Evaluation using Real SAR Image}

To evaluate the proposed method, the real SAR images SAR5, SAR6, SAR7 and SAR8 are considered for experiment. The obtained results compared with existing methods are shown in Figs. 11-14. Non reference quality metrics are calculated for evaluating the quality of despeckled images. Figures 11 and 14 show the result of the proposed method on uncorrelated speckle noise, the distributional behavior of the speckle can be analyzed and known but the degree of noise variance in the image cannot be estimated. Hence, it is a complicated task to despeckle the real noisy SAR image, but even then the proposed method performs better than other methods. Figures 15 and 16 show clear visualization of internal regions in terms of texture and edge features of despeckled real SAR images. $\mathrm{R} 1$ denotes original speckle region and R2, R3, R4, R5, R6 denote the despeckled results from HBSWT [18], GGF-
BNLM [16], CE-CG [17], NSCT-GL [19] and the proposed NSCT-BMAP methods respectively. From this figures it is confirmed that the proposed method preserves maximum edge and texture features than the existing methods. Performance analysis of the proposed NSCTBMAP and existing methods on SAR5, SAR6, SAR7 and SAR8 images using non reference quality metrics are shown in Figs. 17-20. From Figs. 17-20, increased values of MSD, ENL, ESI $\mathrm{H}_{\mathrm{H}}, \mathrm{ESI}_{\mathrm{V}}, \mathrm{SCR}$ and decreased values of NMV and NV confirm that the edges and surface textures are clearly obtained from the proposed method. Apart from evaluating a denoised image based on visual appearance and performance metrics, computational time of the algorithm is also one important factor. The average execution time is calculated for existing and proposed method using synthetic SAR images is shown in Tab. 3.

It can be observed from Tab. 3 that the NSCT-BMAP proposed method takes comparatively less computational

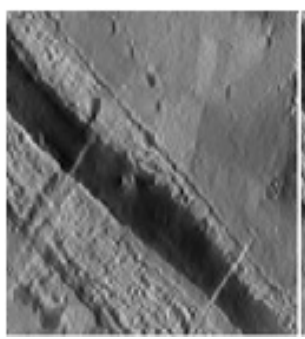

(a)

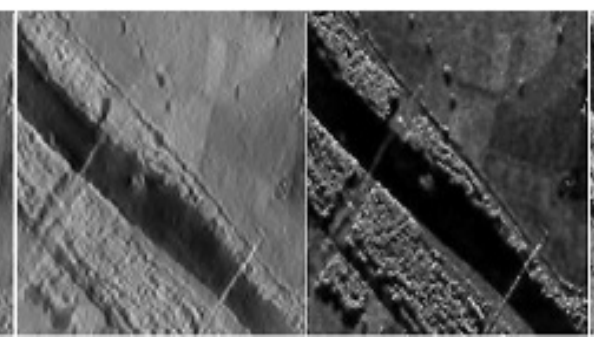

(b) (c)

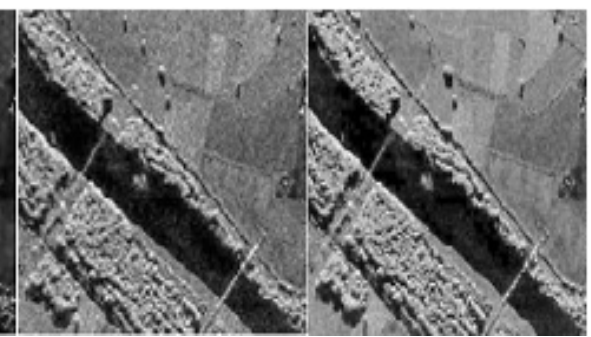

(d) (e)

Fig. 11. Results of the SAR5 image : (a) [18], (b) [16], (c) [17], (d) [19], (e) the proposed method. 


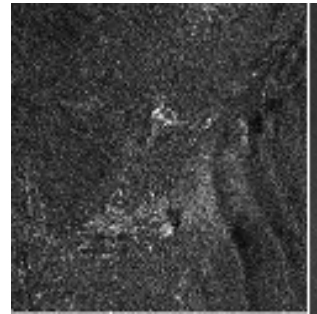

(a)

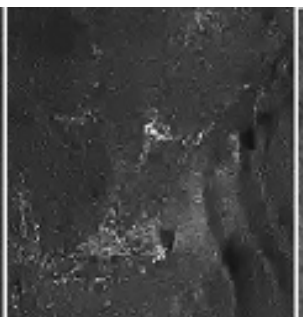

(c)

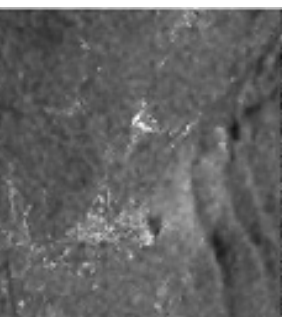

(d)

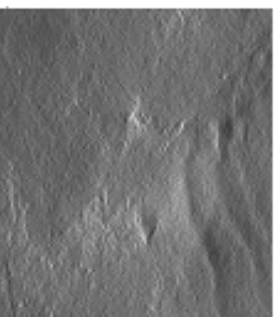

(e)

Fig. 12. Results of the SAR6 image: (a) [18], (b) [16], (c) [17], (d) [19], (e) the proposed method.

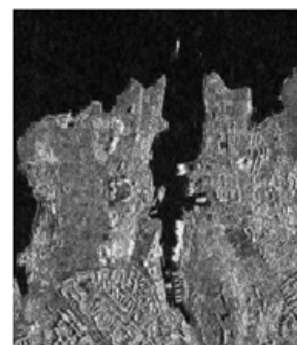

(a)

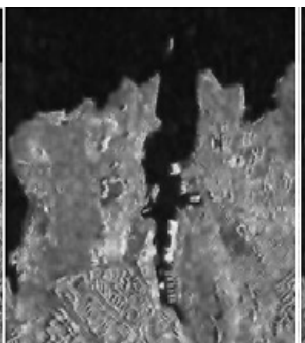

(b)

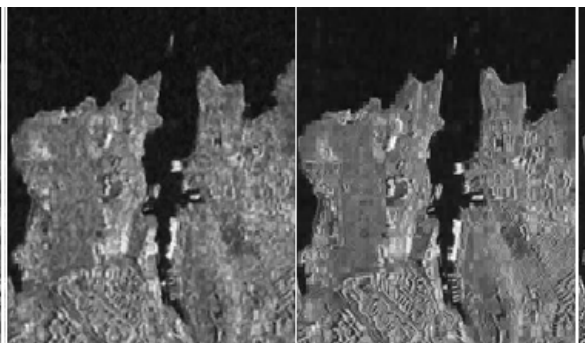

(c)

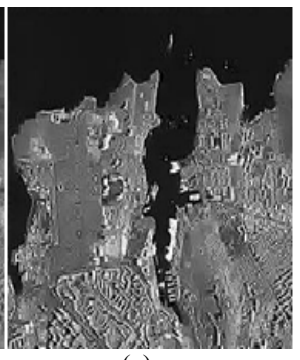

(e)

Fig. 13. Results of the SAR7 image: (a) [18], (b) [16], (c) [17], (d) [19], (e) the proposed method.

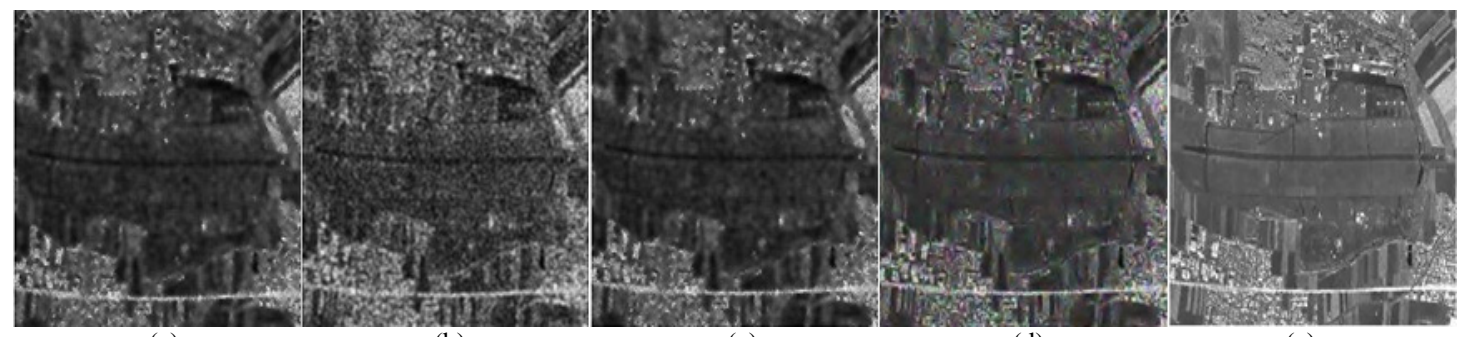

(a)

(b)

(c)

(d)

(e)

Fig. 14. Results of the SAR8 image: (a) [18], (b) [16], (c) [17], (d) [19], (e) the proposed method.

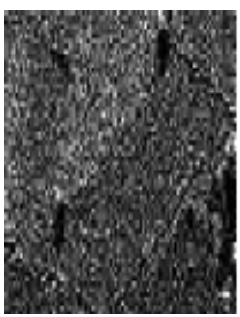

(R1)

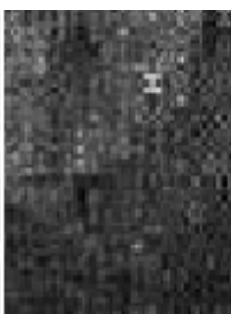

(R2)

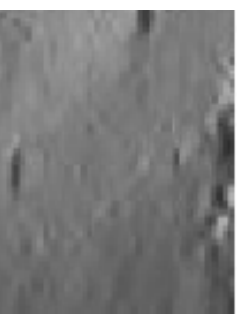

(R3)

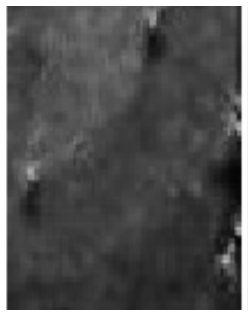

(R4)

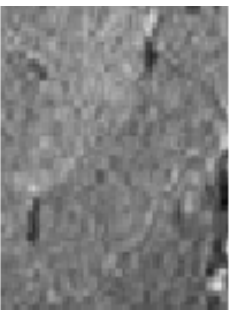

(R5)

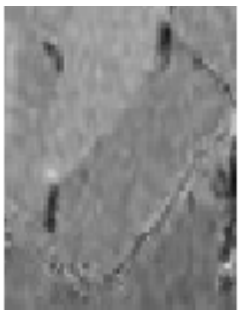

(R6)

Fig. 15. Edges of local region from SAR5 despeckled image:

(R1) Original SAR5, (R2) [18], (R3) [16], (R4) [17], (R5) [19], (R6) the proposed method.

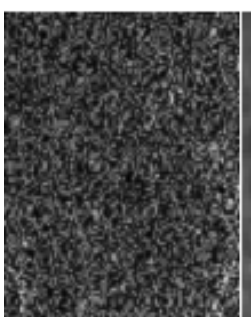

(R1)

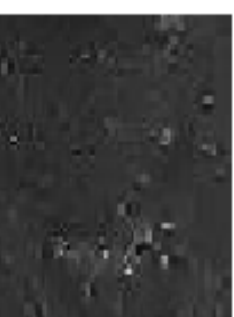

(R3)

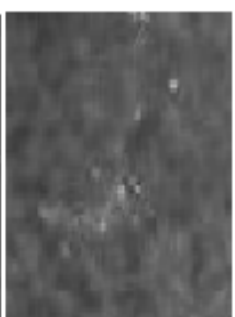

(R4)

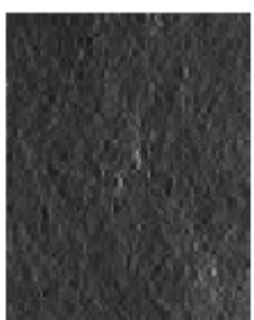

(R5)

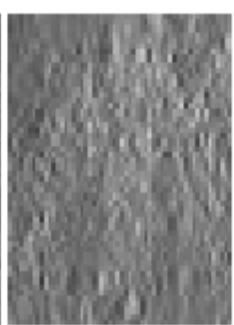

(R6)

Fig. 16. Texture surface of local region from SAR6 despeckled image:

(R1) Original SAR5, (R2) [18], (R3) [16], (R4) [17], (R5) [19], (R6) the proposed method. 


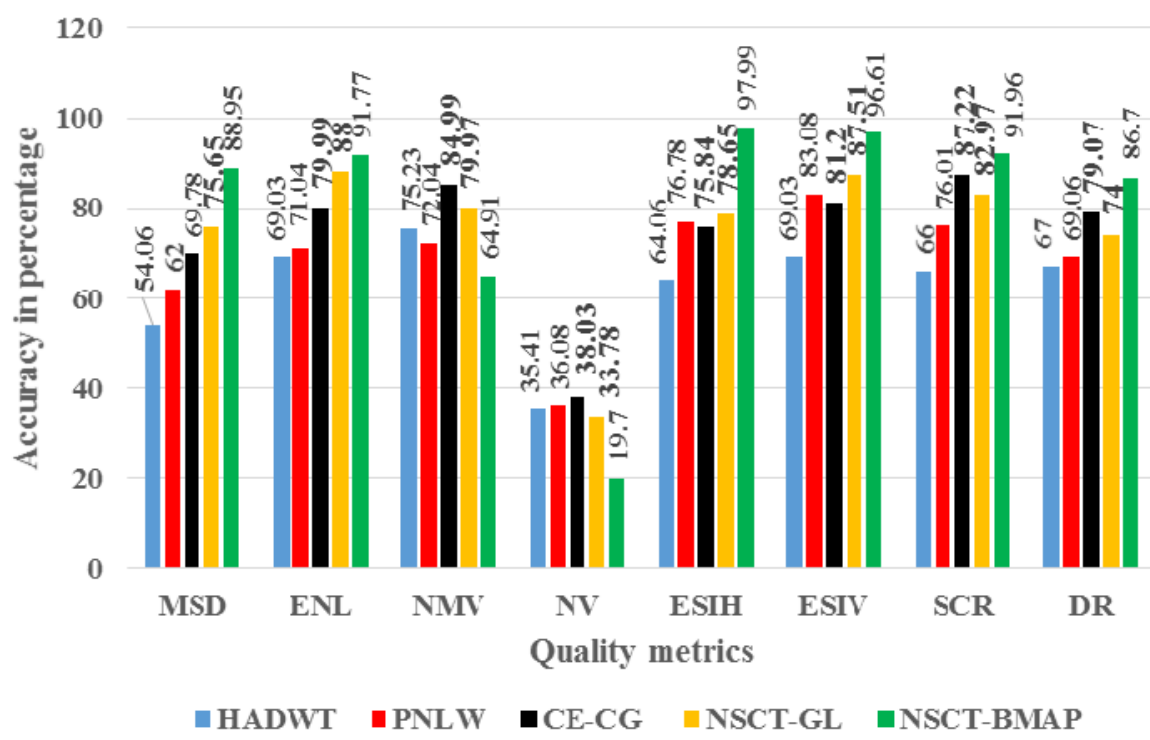

Fig. 17. Performance analysis of the SAR5 image using non- reference quality metrics.

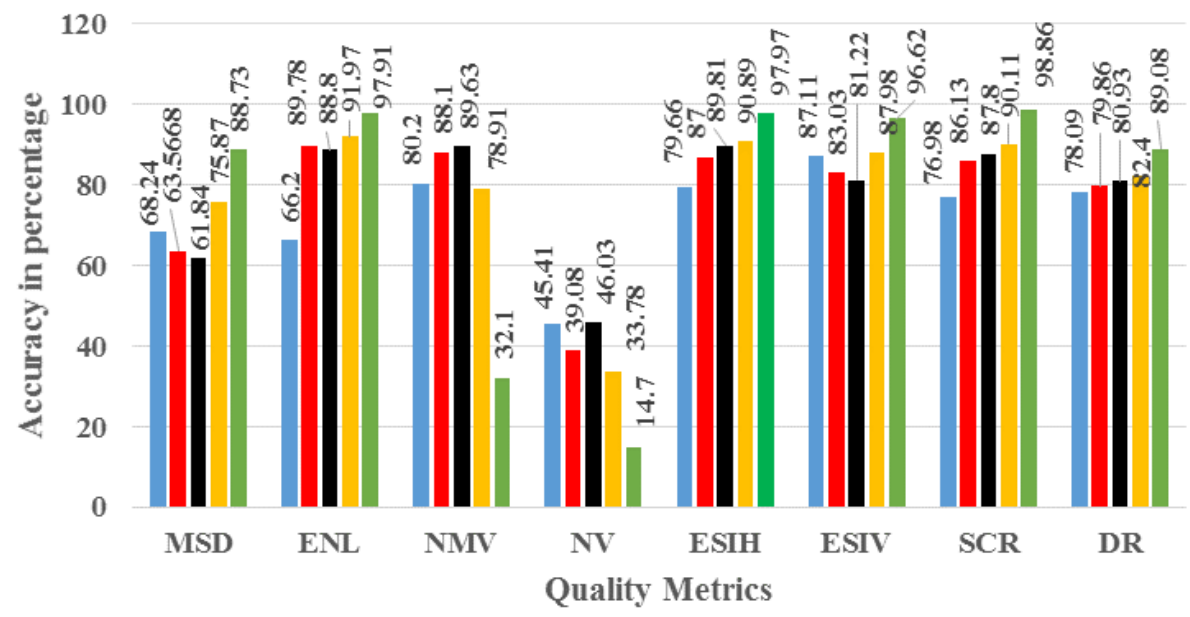

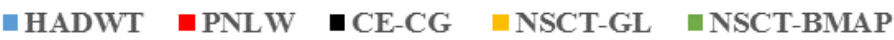

Fig. 18. Performance analysis of the SAR6 image using non- reference quality metrics.

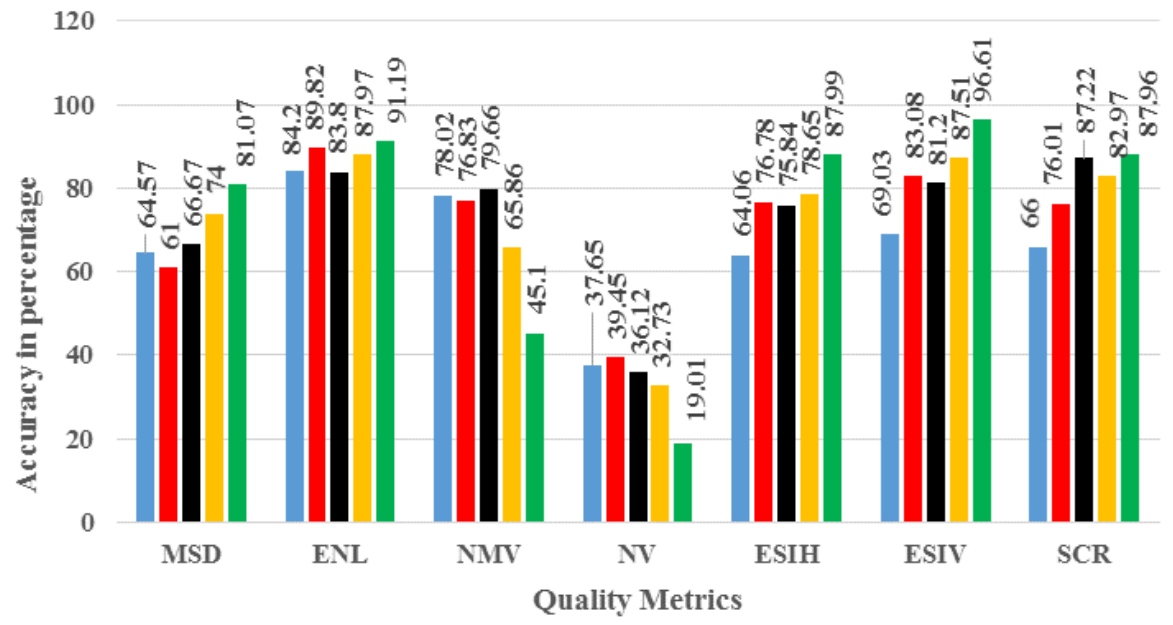

$\square$ HADWT $\square$ PNLW $\square$ CE-CG $\backsim$ NSCT-GL $\backsim$ NSCT-BMAP

Fig. 19. Performance analysis of the SAR7 image using non- reference quality metrics. 


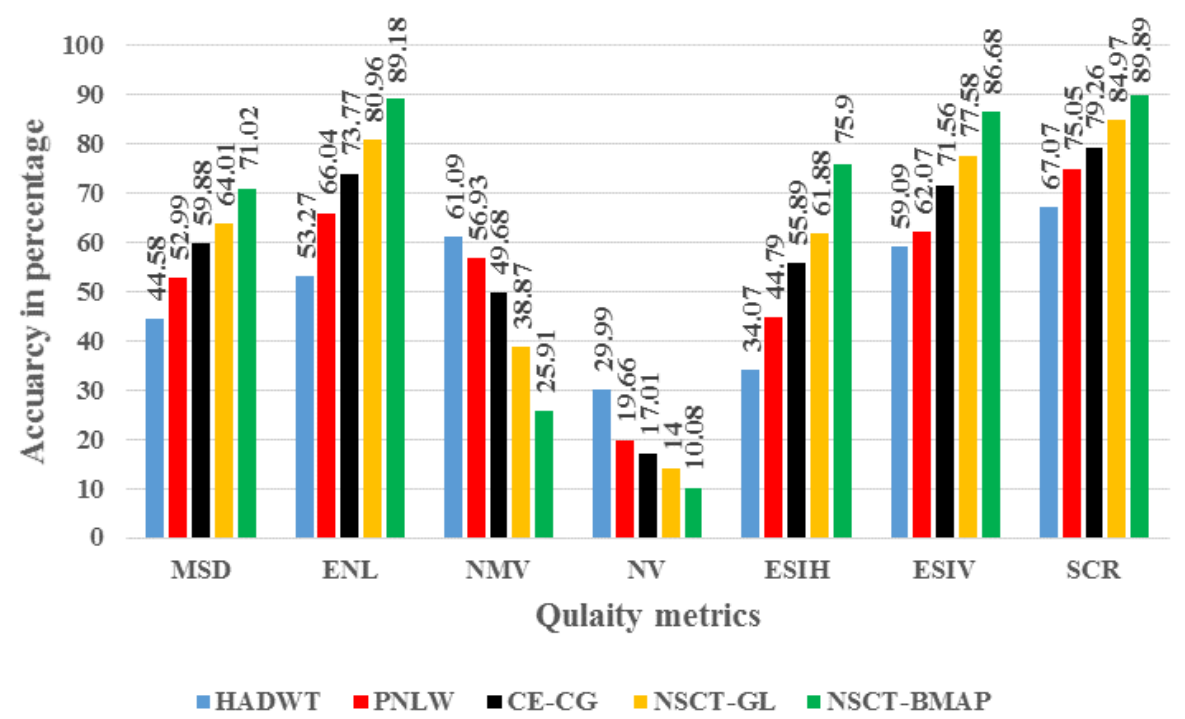

Fig. 20. Performance analysis of the SAR8 image using non- reference quality metrics.

\begin{tabular}{|c|c|c|c|c|c|}
\hline Methods & HBSWT & GGF-BNLM & CE-CG & NSCT- GL & Proposed NSCT-BMAP \\
\hline Time & 3.7810 & 1.0087 & 9.8712 & 2.3456 & 2.3451 \\
\hline
\end{tabular}

Tab. 3. Execution time of the existing and proposed methods in seconds.

time than HBSWT, CE-CG, NSCT- GL but slightly more time than GGF-BNLM. Because GGF-BNLM method is obtained in spatial domain which produces less despeckling results than the other compared methods. So, the less time doesn't affect the proposed method. On comparing Tabs. 1-3, it is confirmed that the results of the proposed algorithm are better than the other recent methods.

\section{Conclusion}

An NSCT-based Bayesian MAP approach for SAR despeckling is presented. NSCT uses sparse representation of images with different scale and dimensionality. SAR images are adequately modeled by the BMAP. Rayleigh distribution and Laplacian distribution are used in modelling the noise and noiseless coefficients, respectively, resulting in accurate statistical estimation results of the despeckled SAR image. Since the original and synthetic SAR images are used, reference and non-reference quality metrics are used for evaluating the quality of the proposed work. The NSCT-BMAP despeckling using the suggested quality measures shows more than $95 \%$ of accuracy in terms of preserving features than the other methods. This method is also more advantageous in other types of SAR images such as L, C, X band, with different resolutions obtained from the different aircrafts and satellites such as RISAT-1, Seasat, RADARSAT-1\&2 etc. This NSCTBMAP method is very useful in detection of ships, eddy current areas and other ocean relevant applications due its higher preservation of edges and texture.

\section{References}

[1] OLIVER, C., QUEGAN, S., Understanding Synthetic Aperture Radar Images. Raleigh, NC: SciTech Publishing, 2004. ISBN: 978-1891121319

[2] DEVAPAL, D., KUMAR, S. S., JOJY, C. Comprehensive survey on SAR image despeckling techniques. Indian Journal of Science and Technology, 2015, vol. 8 , no. 24 . DOI: $10.17485 / \mathrm{ijst} / 2015 / \mathrm{v} 8 \mathrm{i} 24 / 82651$

[3] GLEICH, D., DATCU, M. Gauss-Markov model for waveletbased SAR image despeckling. IEEE Signal Processing Letters, 2006, vol. 13, no. 6, p. 365-368. DOI: 10.1109/LSP.2006.871712

[4] CHEN, G., LIU, X. Contourlet-based despeckling for SAR image using hidden Markov tree and Gaussian Markov models. In IEEE 1st Asian and Pacific Conference on Synthetic Aperture Radar. Huangshan (China), 2007, p. 784-787. DOI: 10.1109/APSAR.2007.4418727

[5] LIAN XUEQIANG, DING XINGHAO, YAN JINGWEN. SAR image despeckling using nonsubsampled Contourlet transform. Chinese Journal of Scientific Instrument, 2008, vol. 29, no. 3, p. 512.

[6] PENG, M., LIU, W. B., ZHANG, G. A SAR image despeckling using self-adaptive threshold function based on NSCT. Journal of Jiamusi University (Natural Science Edition), 2009, vol. 6, p. 005.

[7] CHANG, X., JIAO, L. C., LIU, F., SHA, YH. SAR image despeckling based on the estimation of speckle variance in nonsubsampled contourlet domain. DianziXuebao (Acta Electronica Sinica), 2010, vol. 38, no. 6, p. 1328-1333.

[8] SUN, Q., JIAO, L., HOU, B. Synthetic aperture radar image despeckling via spatially adaptive shrinkage in the nonsubsampled contourlet transform domain. Journal of Electronic Imaging, 2008, vol. 17 , no. 1. DOI: $10.1117 / 1.2841040$ 
[9] MEJÍA MUÑOZ, J. M., DE JESUS OCHOA DOMÍNGUEZ, H., ORTEGA MÁYNEZ, L., et al. SAR image denoising using the non-subsampled contourlet transform and morphological operators. In Mexican International Conference on Artificial Intelligence, 2010, p. 337-347. DOI: 10.1007/978-3-642-167614_30

[10] LANG, S., LIN, C. Y., LIU, J., et al. A comparison of SAR image speckle filters. In Proceeding SPIE (MIPPR 2009: Remote Sensing and GIS Data Processing and Other Applications). 2009, vol. 7498, p. 1-5. DOI: $10.1117 / 12.830946$

[11] QURESHI, S. A., MASOOD, I., HASHMI, M., et al. Noise reduction of electrocardiographic signals using wavelet transforms. Elektronika ir Elektrotechnika, 2014, vol. 20, no. 4, p. 39-42. DOI: $10.5755 /$ j01.eee.20.4.6886

[12] DAS, A. J., TALUKDAR, A. K., SARMA, K. K. An adaptive Rayleigh-Laplacian based MAP estimation technique for despeckling SAR images using stationary wavelet transform. International Journal of Applied Evolutionary Computation (IJAEC), 2013, vol. 4, no. 4, p. 88-102. DOI: 10.4018/ijaec.2013100106

[13] YUE, C., JIANG, W. SAR image denoising in nonsubsampled contourlet transform domain based on maximum a posteriori and non-local constraint. Remote Sensing Letters, 2013, vol. 4, no. 3, p. $270-278$. DOI: $10.1080 / 2150704 X .2012 .723146$

[14] FENG, H. X., HOU, B., JIAO, L. C., et al. SAR image despeckling based on local Gaussian model and MAP in NSCT domain. DianziXuebao (Acta Electronica Sinica), 2010, vol. 38, no. 4, p. $811-816$.

[15] ANANDHI, D., VALLI, S. An algorithm for multi-sensor image fusion using maximum a posteriori and nonsubsampled contourlet transform. Computers \& Electrical Engineering, 2018, vol. 65, p. 139-152. DOI: 10.1016/j.compeleceng.2017.04.002

[16] GOKUL, J., NAIR, M. S., RAJAN, J. Guided SAR image despeckling with probabilistic non local weights. Computers and Geosciences, 2017, vol. 109, p. 16-24. DOI: 10.1016/j.cageo.2017.07.004

[17] MAHAPATRA, D. K., RAY, S. S., ROY, L. P. Maximum a posteriori-based texture estimation by despeckling of SAR clutter amplitude data. IET Image Processing, 2017, vol. 11, no. 8, p. 656-666. DOI: 10.1049/iet-ipr.2017.0344

[18] SINGH, P., SHREE, R. A new homomorphic and method noise thresholding based despeckling of SAR image using anisotropic diffusion. Journal of King Saud University-Computer and Information Sciences, 2017. DOI: 10.1016/j.jksuci.2017.06.006

[19] CHANG, X., JIAO, L., LIU, F., SHA, Y. SAR image despeckling using scale mixtures of Gaussians in the nonsubsampled contourlet domain. Chinese Journal of Electronics, 2015, vol. 24, no. 1, p. 205-211. DOI: $10.1049 /$ cje.2015.01.034
[20] JI, J., LI, Y. An improved SAR image denoising method based on bootstrap statistical estimation with ICA basis. Chinese Journal of Electronics, 2016, vol. 25, no. 4, p. 786-792. DOI: 10.1049 /cje.2016.06.040

[21] TANG, Y., LIU, X. NRDSP: A novel assessment of SAR image despeckling. Neurocomputing, 2017, vol. 249, p. 225-236. DOI: 10.1016/j.neucom.2017.04.002

[22] MASTRIANI, M. New Wavelet-based Superresolution Algorithm for Speckle Reduction in SAR Images. 2006, 8 p. Available at: https://arxiv.org/ftp/arxiv/papers/1608/1608.00270.pdf

[23] NASAPHOTOJOURNAL Laboratories. [Online] Available at: https://www.jpl.nasa.gov

[24] BELGIAN PLATFORM ON EARTH OBSERVATION. [Online] Available at: http://eo.belspo.be/Default.aspx

[25] SANDIA NATIONAL LABORATORIES, AIRBUS DEFENSE AND SPACE. Available at: https://energy.gov/em/sandianational-laboratories and www.intelligence-airbusds.com/en/65satellite-imagery

[26] EUROPEAN SPACE AGENCY (ESA) Available at: http://www.esa.int/ESA

\section{About the Authors...}

Duraipakkiam ANANDHI (corresponding author) completed her B.E and M.E from Annamalai University, Chidambaram, Tamil Nadu, India. Currently, she is pursuing her Ph.D. in the Dept. of Computer Science and Engineering, College of Engineering Guindy, Anna University, Chennai, Tamil Nadu, India. Her research interest covers remote sensing, soft computing, image processing and Bayesian statistics.

Shanmugam VALLI received her B.E. and M.E. degree in Computer Science and Engineering from the Government College of Technology, Coimbatore, Tamil Nadu, India. She got her Ph.D. degree from the Dept. of Computer Science and Engineering, Anna University, Chennai. She is currently a Professor in the Dept. of Computer Science and Engineering, College of Engineering Guindy, Anna University, Chennai, Tamil Nadu, India. Her research interests include parallel computing, compilers, software engineering, image processing, operating system, machine learning and network security. 